\title{
تخمين أعمار الخزانات تحت تاثير المنتوجات النفطية المختلفة
}

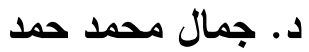 \\ مركز تكنولوجيا الطاقة والطاقات المتجددة - الجامعة التكنولوجية
}

الخلاصة

أستخدمت في هذه الدراسة خمسة محاليل نفطية لقياس معدل تآكل معدن الخزان المخصص لخزن المنتجات النفطية والمصنوع من سبيكة فولاذ منخفض الكاربون هذه المحاليل هي بنزين، ماء مرافق للبنزين، زيت الغاز، ماء مرافق لزيت الغاز ، ماء مرافق للنفط الخام. وتم إختبار ثلاث عينات في كل محلول وأخذ معدل النتائج وذلك للحصول على نتائج أكثر دقة. بينت الدراسة بأن الخطورة ثقع في العمق السفلي للخزان بسبب وجود الماء حيث الوزن النوعي للماء أكبر من الوزن النوعي للبنزين وزيت الغاز • ولذلك لابد من التخلص من الماء المرافق حتى ولو كان على حساب خسارة جزء من المنتوج النفطي وذللك لديمومة عمل الخزان وإطالة عمره.

\section{$\underline{\text { Abstract }}$}

In this study five oil solutions were used to measure the corrosion of the metal tank which was used. This tank is used to hold the oil productions and is made of an alloy called low carbon steel. 
These solutions are Benzene, water associated with benzene, Gas oil, water associated with the gas oil, and water associated with the crude oil.

A three sample were choosing of each solution and the average was taken in order to get a precise result. Also the study clear field that the danger is located in lowest part of the tank because of the existence of the water however, the specific gravity of water is greater than the specific gravity of the benzene and gas oil.

For this reason we have to super water associated even though the loss of a product to increase the duty time and ageing of the used tank.

تعتبر الخزانات النفطية الإنشاءات المعدنية الرئيسية لخزن المنتجات النفطية وتصنع عادة من الفولاذ المقاوم للتآكل [1]. إن المشكلة الرئيسية التي تتعرض لها الخزانات النفطية هي التفاعل الذي يحدث مابين معدن الخزان والمادة النفطية المخزنة فيه وخاصة إذا إحتوت هذه المادة على الماء المرافق، وينتج عن ذللك تآكل معدن الخزان والذي يستمر حتى يصل إلى حدود معينة لايمكن للخزان أن يتحملها مما يؤدي إلى حدوث ثقوب في جدران الخزان تؤدي إلى تدفق المادة النفطية على جدران الخزان والذي يسبب الحرائق ويؤدي إلى تلف الخزان وملحقاته وهدر المادة النفطية المخزنة والني هي عبارة عن مزيج من مواد هيدروكاربونية مكونة بالإساس من مركبات تحتوب على الكاربون 
والهيدروجين وبعض العناصر المختلطة مع الهيدروكاربونات مثل الكبريت والنيتروجين والاوكسجين بالأضافة إلى عناصر فلزية نوجد بنسب بسيطة كالحديد والنيكل والزرنيخ والفناديوم. يتم تقييم دعدل التآكل بعدة طرق وهي: 1

تعتمد هذه الطريقة على كمية المادة المفقودة من العينة بعد تعرضها لوسط التآكل ويعبر عن معدل التآكل بهذه الطريقة بمقدار الوزن المفقود من وحدة المساحة في وحدة الزمن [2]، هذا عندما يكون معدل الفقدان بالوزن ثابتاً خلال فترة التعريض ومتجانساً خلال سطح المعدن، ولهذا التعبير وحدات عديدة منها (mdd) عندما يكون الوزن بالملغرام (milligram) والزمن بالايام (day) والمساحة السطحية بالدسمز المربع (decimeter). وهناك قياس (mpy) حيث يرمز للحرف m الفقدان بالابعاد مقاساً بالمل (1/1000 إنج) والحرف p per) والحرف y الى الزمن بالسنوات (year).

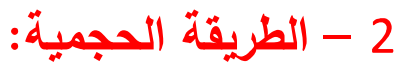

عند حدوث عملية التآكل للمعادن تتحرر غازات وتتطلق الى الجو الخارجي بفعل التفاعل الكيميائي الذي يحدث، ويمكن أن يتم خلال هذه العملية إمتصاص غازات من الجو الخارجي، وبذلك بمكن 
تحديد معدل التآكل من خلال قياس حجم هذا الغاز حيث أن حجماً مولياً من الغاز المتحرر أو الممتص يؤكسد n(g-Eqv غراماً مكافئاً من المعدن [3]، وبالتالي فأن وزن المعدن المتآكل يكون

$$
g=(n v \times A) /\left(V_{m o l} \times Z\right)
$$

g : حجم الغاز المنطلق (الممتص) المقاس

V

A : - (الوزن الذري للمعدن A

Z : تكافؤ المعدن

ومن خلال معرفة سطح المعدن المختبر وبواسطة العلاقة أعلاه يمكن حساب سرعة تآكل المعدن.

3 - طريقة تيار التآكل الكهروكيميائي:

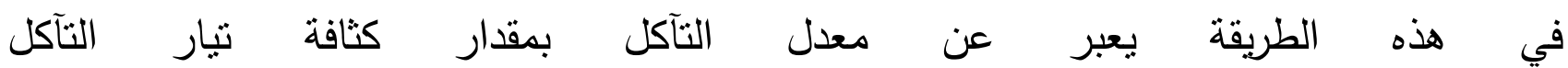
ولهذا وحدات عديدة اهمها (corrosion current density, I I corr) معدل التآكل المعبر عنه بكثافة تيار التآكل الى تعبير آخر بوحدات اخرى مثل القانون التالي [3]:

$$
R(m p y)=0.13 \times I_{\text {corr }} \times c / p
$$


ثم إجراء التجارب على عينات من نفس نوع معدن الخزانات، وإجراء تحليل للعناصر السبائكية للعينة وكانت مايلي : 


\begin{tabular}{|r|r|r|r|r|r|r|}
\hline S & P & Mn & Si & c & Fe & \\
\hline 0.0082 & 0.012 & 1.19 & 0.334 & 0.165 & Rim & \\
\hline
\end{tabular}

3 - أوساط الإختبار : -

تم إجراء تجارب التآكل في أوساط مختلفة مشابهة لتنلك التي تتعرض لها الخزانات النفطية أثثاء العمل

$$
\text { زيت الغاز }
$$

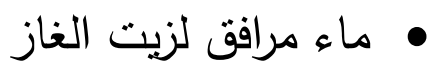

ماء مرافق للنفط الخام

$$
4
$$

تمت التجارب على عينات في الاوساط المذكورة أعلاه لغرض معرفة معدل التاكل في تلاك الأوساط، وتم اعتماد الطريقة الكهروكيميائية لتحديد قيمة تيار التآكل، لان هذه الطريقة تعطي نتائج أكثر دقة من الطرق الأخرى الوزنية والحجمية [5]. 


\section{النتائج والمناقشتة}

تم من خلال التجارب العملية الحصول على خمس مخططات تبين العلاقة بين كل من الجهد وتيار التاكل وذلك للحالات الخمس المختبرة وهي كما يلي:

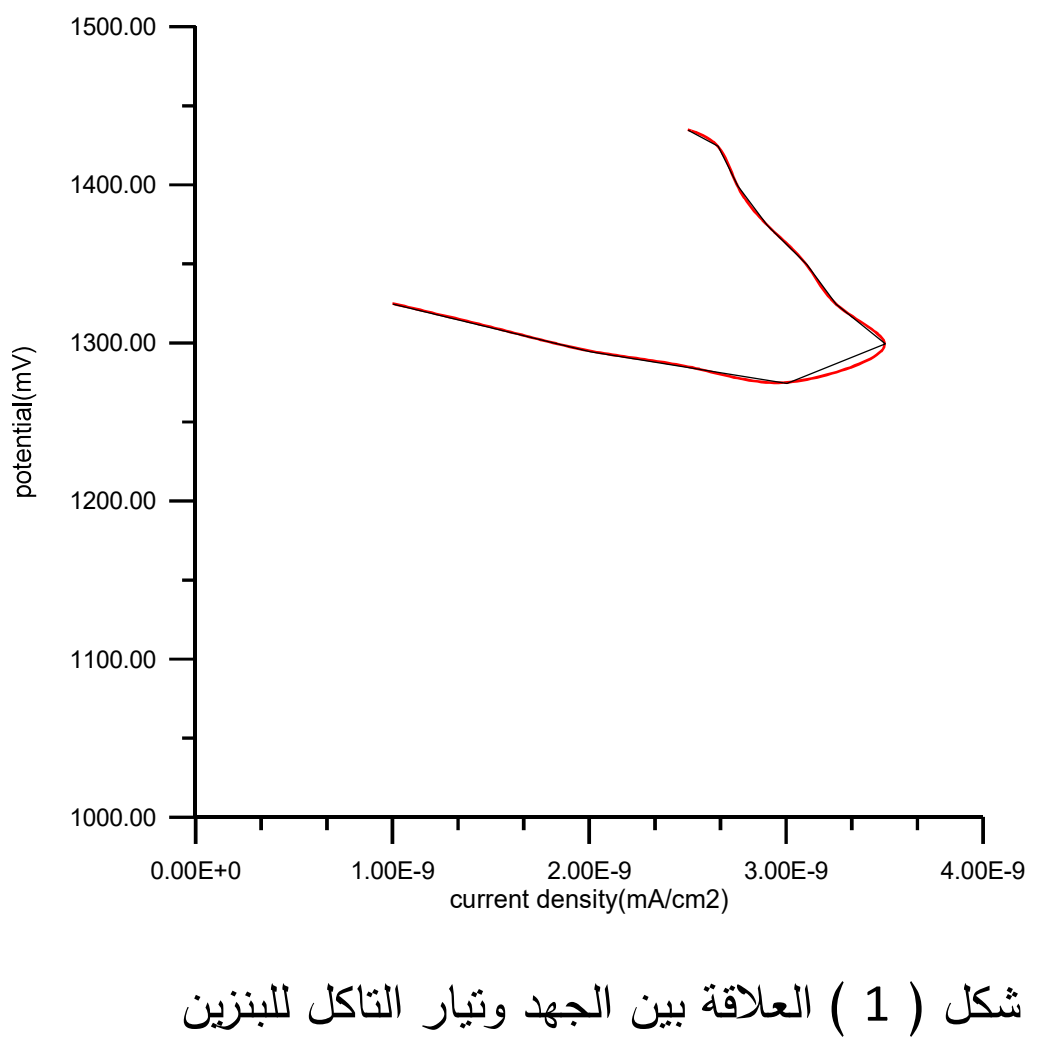




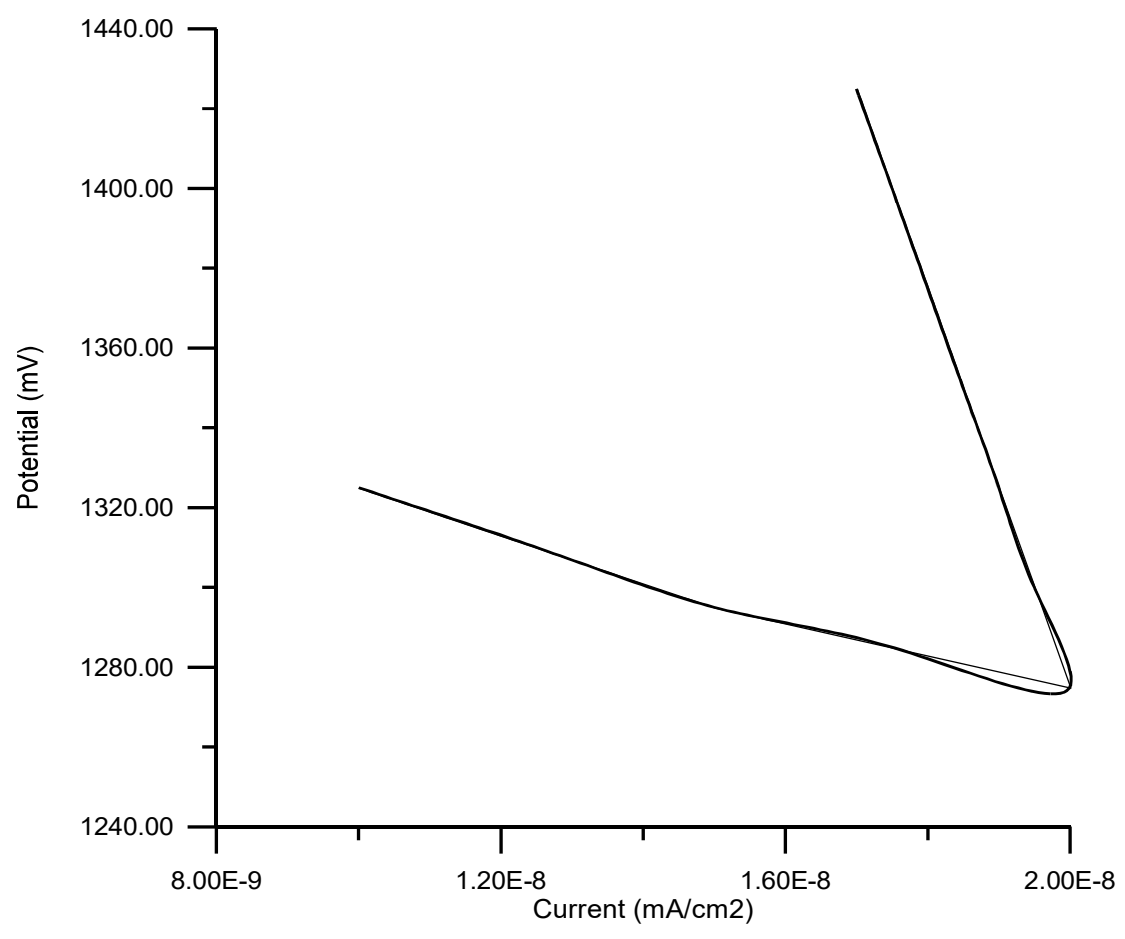

شكل ( 2 ) العلاقة بين الجهد وثيار التاكل لزيت الغاز

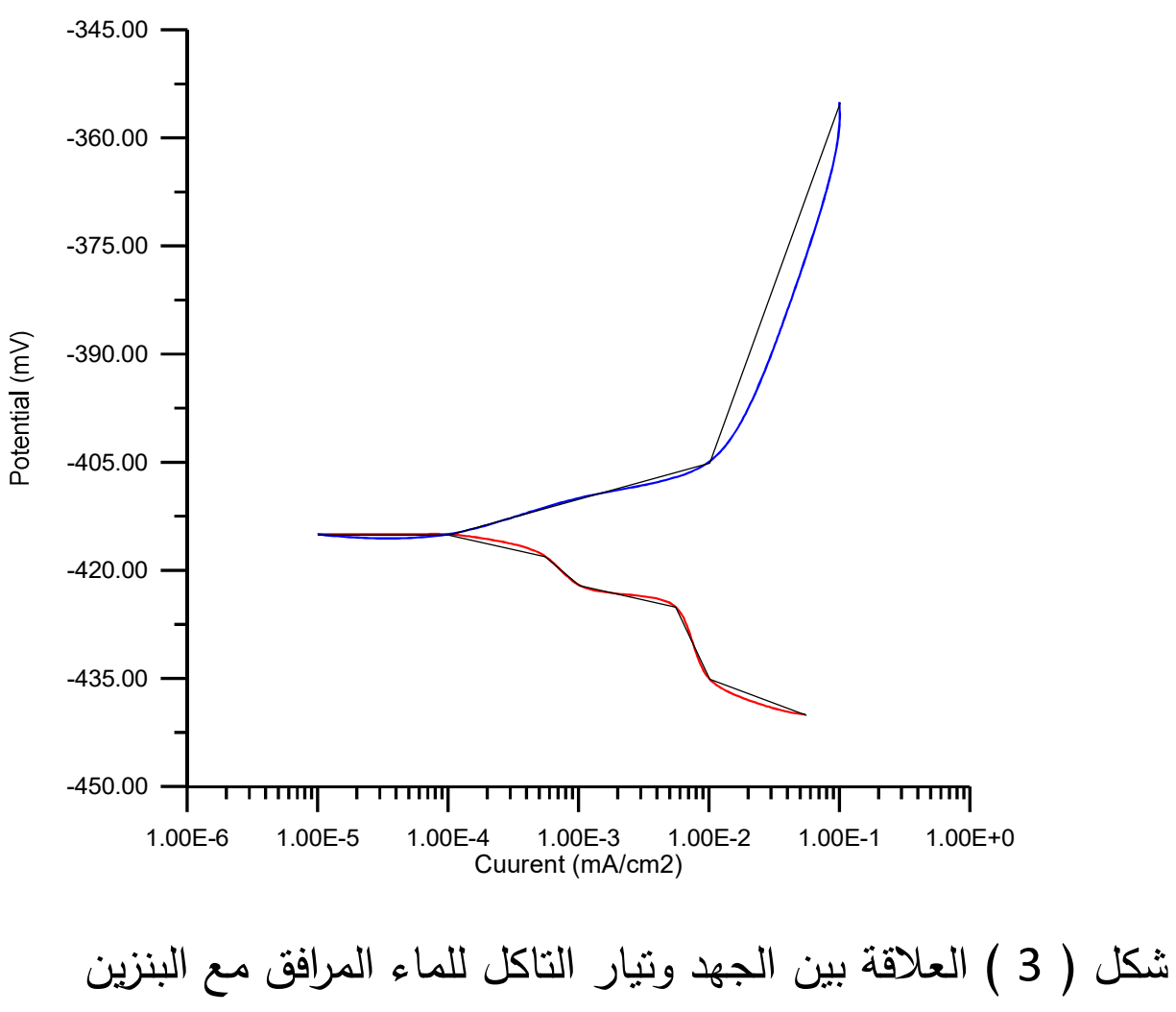




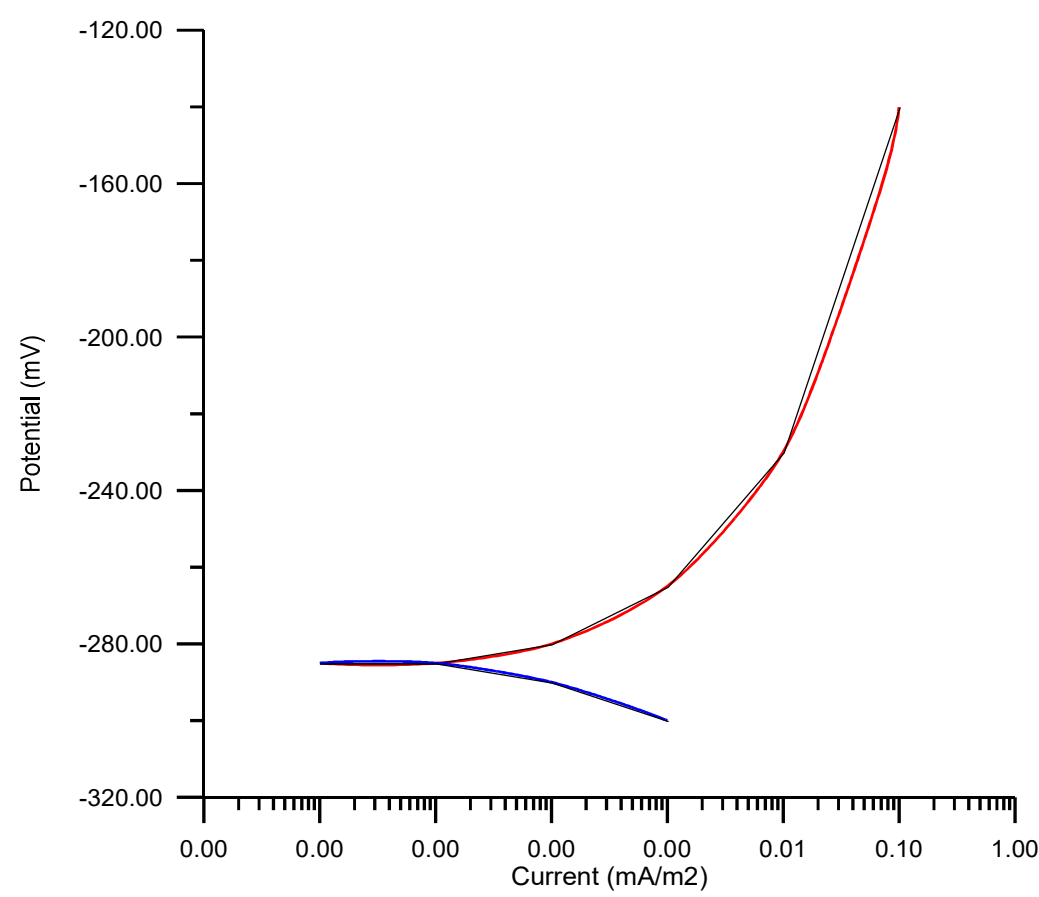

شكل ( 4 ) العلاقة بين الجهد وتيار التاكل للماء المرافق لزيت الغاز

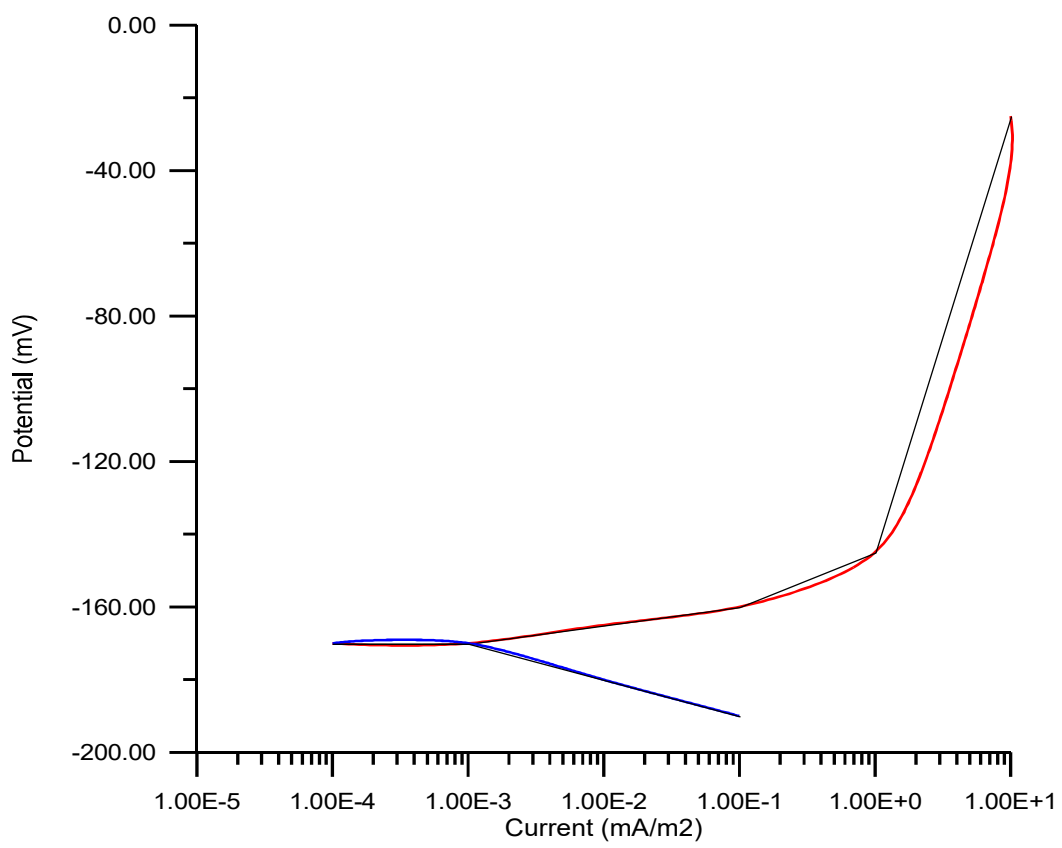

شكل ( 5 ) العلاقة بين الجهد وتيار التاكل للماء المرافق مع النفط الخام 
وقد بينت النتائج بان معدلات الناكل كانت كما يلي

$1.35 \times 10^{-8} \mathrm{~mm} /$ year بنزين

$1.89 \times 10^{-7} \mathrm{~mm} /$ year

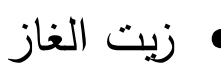

$0.687 \mathrm{~mm} /$ year

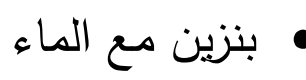

0.236 mm/year مبت الغاز مع الماء

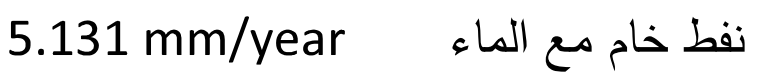

واذا علمنا بان معدل التاكل المسموح به للخزانات التي تحوي مواد نفطية هو mm (3 - 1.5) حسب المواصفة الاميريكية API650 [1]، فانه يمكن حساب اعمار الخزانات والتي كانت كما يلي
$\left(11 \times 10^{7}-22 \times 10^{7}\right)$ year بنزين
$\left(8 \times 10^{6}-16 \times 10^{6}\right)$ year

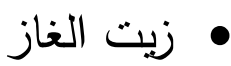
$(2.2-4.4)$ year بنزين مع الماء
$(6.3-12.6)$ year
زيت الغاز مع الماء
$(0.3-0.6)$ year
• نفط خام مع الماء 


\section{الاستنتاجات : - الإن}

لوحظ بان اخطر منطقة في الخزان هي المنطقة السفلية وذلك لوجود الماء حيث الوزن النوعي له اكبر من الوزن النوعي للبنزين او زيت الغاز [6]، ولذلك لابد من زيادة سمك الطوق السفلي للتغلب على مشكلة التاكل وبالتالي زيادة عمر الخزان. ولابد من التخلص من الماء الزائد المرافق للمنتوج النفطي حتى لو كان على حساب خسارة جزء من المنتوج النفطي. ان وجود الماء في اسفل الخزان قد يسبب حرائق بسبب التبخر الذي يحدث حيث ان حجم واحد من الماء يتبخر ليعطي (1750) حجم من البخار تقريبا وان هذا البخار يندفع الى الاعلى حاملا معه كميات من المنتج النفطي قاذفا به الى مسافات بعيدة.

ان زيادة التاكل عن الحد المسموح به قد يؤدي الى وجود نقاط ضعف على جدران الخزان وتزداد خطورته عند ارتفاع درجة الحرارة، لذلك لابد من المراقبة الدائمة وعزل الخزانات عند وصول مقدار

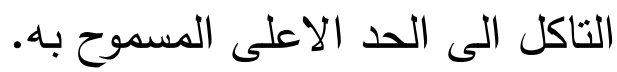
ان وجود فوهات تصريف سفلية في الخزان لطرد المياه المرافقة امر في غاية الاهمية للتخلص من مشاكل التاكل والحرائق بشكل كبير , بالاضافة الى منظومة الحماية الكاثودية والتي تقلل من

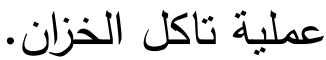


1- API Standard 620," Design and construction of Large, Welded, Low pressure storage tanks", Edition 1998,US.

2- ASM HANDBOOK - Corrosion : Fundamentals, Testing, and Protection - Volume 13A -2003.

3- ASM HANDBOOK - Corrosion : Fundamentals , Testing, and Protection - Volume 13 -1992.

4- Corrosion Industrial Problems, Treatment, and Control Techniques. Edited by V. ASHWORTH Global Corrosion Counsultants, UK. Published on behalf of the Kuwait Foundation for the Advancement of Sciences (KFAS), Volume 2, Proceeding on the $1^{\text {st }}$ Arabian Conference on Corrosion Kuwait, 1984.

5- Technical researches achieved by Corrosion section at AFPC, Ali. Y - Nov.2002, includes: pipeline corrosion, introduction to corrosion , corrosion mechanism.

6- Corrosion control in petroleum production (an official NACE publication 1999).

7- corrosion basics: an introduction National Association of Corrosion Engineering, Houston, Texas, 1984. 